\title{
Mycobacterium chelonae necrotizing pneumonia after allogeneic hematopoietic stem cell transplant: report of clinical response to treatment with tigecycline
}

\author{
E. Peres, Y. Khaled, O.I. Krijanovski, S. Mineishi, J.E. Levine, D.R. Kaul, \\ J. Riddell IV. Mycobacterium chelonae necrotizing pneumonia after \\ allogeneic hematopoietic stem cell transplant: report of clinical \\ response to treatment with tigecycline. \\ Transpl Infect Dis 2009: 11: 57-63. All rights reserved
}

\begin{abstract}
We present a case of progressive Mycobacterium chelonae ssp. chelonae necrotizing pneumonia after hematopoietic stem cell transplantation (HSCT) in the presence of chronic graft-versus-host disease. The patient failed to respond to standard combination therapy with multiple agents and developed resistance to most drugs over the course of treatment. Tigecycline, a new glycylcycline antimicrobial agent with in vitro activity against $M$. chelonae, was then used with a clinical response to treatment. To our knowledge, this is the first reported case demonstrating tigecycline to have a degree of clinical effectiveness to treat refractory pulmonary infection with $M$. chelonae in an HSCT recipient.
\end{abstract}

\author{
E. Peres ${ }^{1}$, Y. Khaled ${ }^{1}$, O.I. Krijanovski', \\ S. Mineishi ${ }^{1}$, J.E. Levine ${ }^{1}$, D.R. Kaul ${ }^{2}$, \\ J. Riddell IV ${ }^{2}$ \\ ${ }^{1}$ Blood and Marrow Transplant Program, University of \\ Michigan Comprehensive Cancer Center, and ${ }^{2}$ Division of \\ Infectious Diseases, University of Michigan Medical School, \\ Ann Arbor, Michigan, USA \\ Key words: stem cell transplant; non-tuberculous \\ mycobacterium; pneumonia; tigecycline; Mycobacterium \\ chelonae \\ Correspondence to: \\ Edward Peres, MD, Division of Hematology and Oncology, \\ University of Michigan Comprehensive Cancer Center, \\ 5303 Cancer Center, 1500 E. Medical Center Drive, SPC 5941, \\ Ann Arbor, MI 48109-5941, USA \\ Tel: +7349368785 \\ Fax: + 7349368788 \\ E-mail: edwardpe@umich.edu
}

Received 10 July 2008, accepted for publication 21 July 2008

DOI: 10.1111/j.1399-3062.2008.00351.x

Transpl Infect Dis 2009: 11: $57-63$
Hematopoietic stem cell transplantation (HSCT) is an effective treatment for a wide variety of hematological malignancies and non-malignant conditions. Opportunistic infections remain an important cause of mortality in this patient population. This is especially true in patients who develop acute and chronic graft-versus-host disease (GVHD) and require immunosuppressive therapy to treat this complication of HSCT. In addition to fungal, bacterial, and viral infections in HSCT recipients, non-tuberculous mycobacterial (NTM) infections have also been rarely reported (1-4).

The overall incidence of NTM infections appears to be increasing. In one recent study, up to $2.9 \%$ of patients who were HSCT recipients developed pulmonary infection from NTM (1). Some rapidly growing NTM species, such as Mycobacterium abscessus and Mycobacterium chelonae, present unique challenges with regards to diagnosis, as it is often difficult to distinguish between true infection and colonization. Treatment is often complicated by therapyrelated toxicity, as well as drug interactions (5). Because of the uncommon nature of $M$. chelonae pulmonary infection in the setting of HSCT, data on mortality is difficult to interpret and is limited to case reports. In general, treatment of $M$. chelonae is successful in many cases (6) as compared with $M$. abscessus, which typically does not respond well to medical therapy $(7,8)$.

We report the case of a patient with chronic GVHD after HSCT who developed refractory, progressive $M$. chelonae ssp. chelonae necrotizing pneumonia despite cessation of all immune suppressive therapy. Treatment with prolonged combination antimicrobial therapy, as well as adjunctive immunomodulatory agents, had little effect on the patient's clinical course. Repeated courses of treatment with tigecycline demonstrated clinical benefit. 


\section{Case report}

A 34-year-old woman was diagnosed with T-cell acute lymphoblastic leukemia and underwent a conditioning regimen consisting of cyclophosphamide and total body radiation followed by a $5 / 6$ human leukocyte antigen-matched allogeneic related peripheral blood SCT from her father in March 2002. GVHD prophylaxis consisted of tacrolimus and mini-methotrexate. She went on to develop chronic GVHD involving the lung, skin, and sclera 3 months later. She was treated with tacrolimus, CellCept (mycophenolate mofetil), and $16 \mathrm{mg}$ of Medrol (methylprednisolone) that was tapered over a period of 6 months. One year later she again required Medrol for GVHD therapy that was tapered from $60 \mathrm{mg} /$ day over the course of 12 months until June 2004.

In August 2004, she developed a chronic cough and was found to have a 3 -cm cavitary left upper lobe nodule on computed tomography (CT) scan. Bronchoscopy with bronchoalveolar lavage (BAL) was non-diagnostic. She then presented in April 2005 with ongoing respiratory symptoms consisting of a non-productive cough and dyspnea. Radiological evaluation revealed new cavitary pulmonary nodules (Fig. 1). The acid-fast bacilli (AFB) smear from BAL fluid was positive with a moderate number of organisms observed. Fungal culture yielded Candida krusei and probable Fusarium or Acremonium that could not be further characterized, which were considered colonizing organisms. Bacterial culture yielded oral flora and the Pneumocystis jirovecii polymerase chain reaction was negative. When the AFB culture yielded $M$. chelonae ssp. chelonae with transbronchial biopsy specimens that demonstrated granulomatous inflammation, she was treated with a combination of cefoxitin $6 \mathrm{~g} /$ day for 4 weeks and amikacin $400 \mathrm{mg}$ twice a day for 2 weeks (discontinued after the development of tinnitus). Azithromycin $500 \mathrm{mg} /$ day and levofloxacin $500 \mathrm{mg} /$ day were also administered and subsequently continued long term. Her immunosuppressive therapy was tapered. She was later initiated on extracorporeal photopheresis (ECP) therapy as a single modality of treatment for her chronic GVHD and was able to taper her corticosteroid therapy.

Despite antimicrobial treatment, she developed progressive infection with an enlarging pulmonary mass and multiple cavitating pulmonary nodules (Fig. 2). Because she developed tinnitus after receiving amikacin, treatment with aminoglycosides was not repeated. Subsequent therapy included treatment with meropenem, imipenemcilastin, linezolid, moxifloxacin, clarithromycin, and inhaled tobramycin in various combinations for periods of 6 weeks (Table 1). She was maintained on treatment with oral moxifloxacin and clarithromycin in an effort to prevent

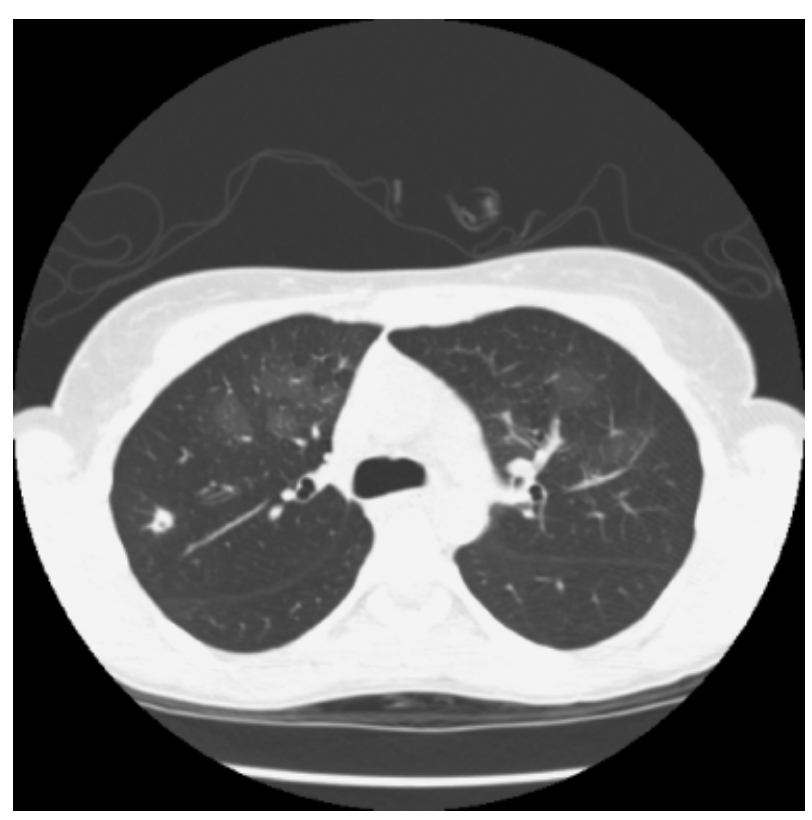

Fig. 1. Presenting chest computed tomography scan in April 2005 with small cavitating nodule in the right upper lobe.

further relapse of the infection. Follow-up imaging demonstrated improvement in some areas with the development of new infection in others (Fig. 3). Repeated bronchoscopy and sputum specimens yielded $M$. chelonae on multiple occasions. Serial susceptibility testing of the M. chelonae isolate demonstrated the progressive development of resistance to all tested antimicrobials with the exception of clarithromycin and tigecycline (Table 2). Despite therapy, she had relapse of infection in areas of previously improved disease (Fig. 4). Because of her underlying poor pulmonary function from GVHD, she was not a candidate for surgical resection of the involved lung tissue. She was not considered for lung transplant because of the risk for subsequent infection of the transplanted organ with $M$. chelonae from the upper respiratory tract.

Adjunctive therapy with granulocyte-macrophage colony-stimulating factor (GM-CSF) to enhance macrophage function and non-steroidal anti-inflammatory drugs (NSAIDs) to modify prostaglandin $\mathrm{E}_{2}$ activity had no affect on the course of her infection. Flow cytometry did not disclose any significant deficiency in lymphocyte subsets (Table 3). She then was treated with 4 separate 6 -week courses of tigecycline when recurrent symptoms of worsening cough, dyspnea, and overall decline in functional status occurred. From 1 to 2 weeks after the initiation of tigecycline, these symptoms significantly improved; however, radiographic resolution of the infection was not achieved (Fig. 4). 


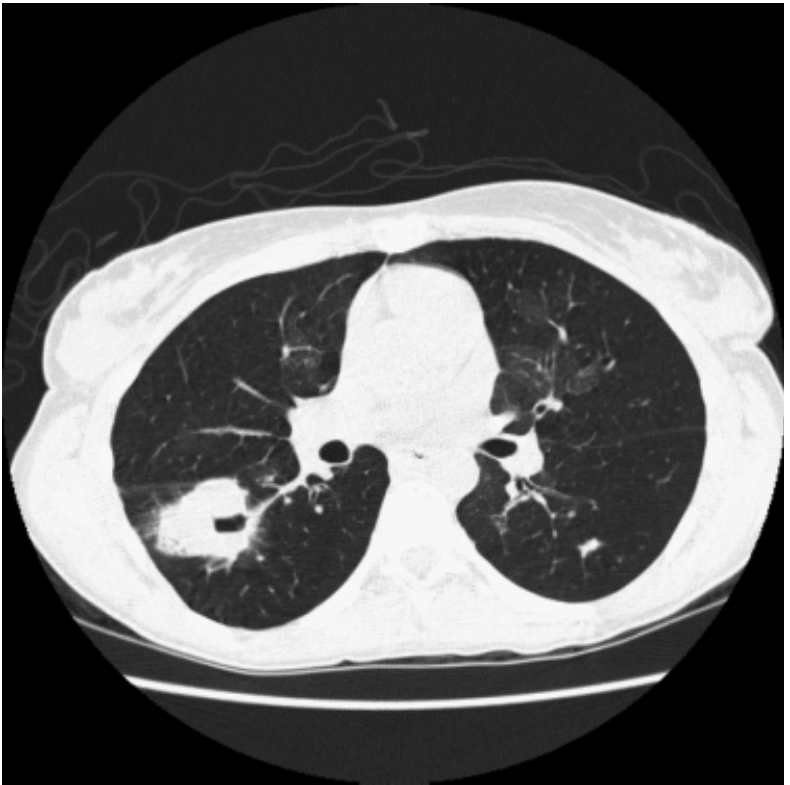

Fig. 2. Chest computed tomography scan in May 2006 with an increasing cavitary mass in the right upper lobe despite treatment.

\section{Discussion}

Our report illustrates a difficult-to-manage case of chronic necrotizing pulmonary infection with M. chelonae ssp. chelonae in the setting of allogeneic HSCT with prior GVHD of

\section{Summary of antimicrobial therapy}

\begin{tabular}{|c|c|}
\hline Date & Antimicrobials and duration \\
\hline $5 / 2005$ & $\begin{array}{l}\text { Cefoxitin } 6 \mathrm{~g} / \mathrm{d} \times 4 \text { weeks, amikacin } 400 \mathrm{mg} \mathrm{q} 12 \mathrm{~h} \times \\
2 \text { weeks, azithromycin, levofloxacin }\end{array}$ \\
\hline $8 / 2005$ & $\begin{array}{l}\text { Cefoxitin } 12 \mathrm{~g} / \mathrm{d} \times 6 \text { weeks, Linezolid } \times 4 \text { weeks, } \\
\text { azithromycin, levofloxacin }\end{array}$ \\
\hline $1 / 2006$ & $\begin{array}{l}\text { Meropenem } 1 \mathrm{gq} 8 \mathrm{~h} \times 6 \text { weeks, Linezolid } \times 6 \text { weeks, } \\
\text { azithromycin, levofloxacin }\end{array}$ \\
\hline $5 / 2006$ & $\begin{array}{l}\text { Imipenem-cilastin } 1 \mathrm{~g} \mathrm{q} 6 \mathrm{~h} \times 6 \text { weeks, tigecycline } \times \\
6 \text { weeks, linezolid } \times 4 \text { weeks, clarithromycin, levofloxacin }\end{array}$ \\
\hline $8 / 2006$ & $\begin{array}{l}\text { Cefoxitin } 12 \mathrm{~g} / \mathrm{d} \times 6 \text { weeks, tigecycline } \times 6 \text { weeks, } \\
\text { clarithromycin, gatifloxacin }\end{array}$ \\
\hline $11 / 2006$ & $\begin{array}{l}\text { Imipenem-cilastin } 1 \mathrm{~g} \mathrm{IV} \mathrm{q} 6 \mathrm{~h} \times 6 \text { weeks, inhaled amikacin } \\
100 \mathrm{mg} / 3 \mathrm{~mL} \text { saline nebulized t.i.d. } \times 4 \text { weeks, } \\
\text { clarithromycin, gatifloxacin }\end{array}$ \\
\hline $5 / 2007$ & Tigecyline $\times 6$ weeks, clarithromycin, gatifloxacin \\
\hline $8 / 2007$ & Tigecyline $\times 4$ weeks, clarithromycin, gatifloxacin \\
\hline $1 / 2008$ & Tigecyline $\times 6$ weeks, clarithromycin, gatifloxacin \\
\hline $6 / 2008$ & Tigecyline $\times 6$ weeks, clarithromycin, gatifloxacin \\
\hline
\end{tabular}

Table 1

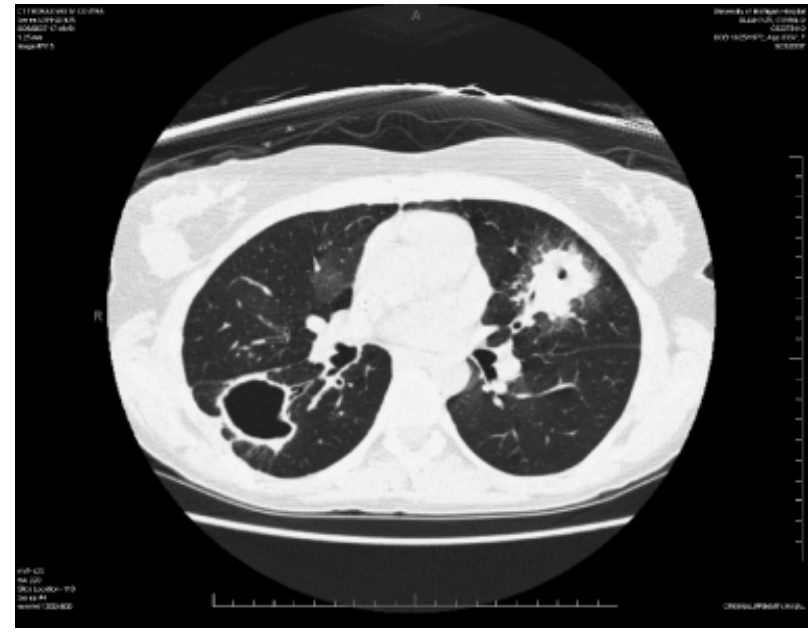

Fig. 3. Chest computed tomography scan in May 2007 demonstrating improvement in right upper lobe infection, but the development of left upper lobe disease.

\section{Mycobacterium chelonae susceptibility pattern over time}

\begin{tabular}{|c|c|c|c|c|c|c|}
\hline \multirow[b]{3}{*}{ Antibiotics } & \multicolumn{6}{|c|}{ Date of specimen acquisition } \\
\hline & \multicolumn{2}{|l|}{$7 / 21 / 05$} & \multicolumn{2}{|l|}{$5 / 19 / 06$} & \multicolumn{2}{|l|}{$8 / 22 / 06$} \\
\hline & $\begin{array}{l}\text { MIC } \\
(\mu \mathrm{g} / \mathrm{mL})\end{array}$ & Int & $\begin{array}{l}\mathrm{MIC} \\
(\mu \mathrm{g} / \mathrm{mL})\end{array}$ & Int & $\begin{array}{l}\text { MIC } \\
(\mu \mathrm{g} / \mathrm{mL})\end{array}$ & Int \\
\hline Amikacin & 8 & $\mathrm{~s}$ & $\leq 8$ & $\mathrm{~S}$ & 64 & $\mathrm{R}$ \\
\hline Cefoxitin & 32 & I & 16 & $\mathrm{~S}$ & 64 & I \\
\hline Ciprofloxacin & 8 & $\mathrm{R}$ & 4 & $\mathrm{R}$ & 2 & 1 \\
\hline Doxycycline & $>64$ & $\mathrm{R}$ & $>32$ & $\mathrm{R}$ & $>32$ & $\mathrm{R}$ \\
\hline Gatifloxacin & 8 & 1 & 4 & 1 & 8 & 1 \\
\hline Imipenem & - & - & 8 & $1^{2}$ & $>16$ & $\mathrm{R}^{2}$ \\
\hline Meropenem & - & - & 16 & $\mathrm{R}^{3}$ & $>16$ & $\mathrm{R}^{3}$ \\
\hline Linezolid & 4 & $\mathrm{~S}$ & 32 & $\mathrm{R}$ & 32 & $\mathrm{R}$ \\
\hline Minocycline & - & - & 6 & 1 & - & - \\
\hline Sulfisoxazole & 64 & $\mathrm{R}$ & $6^{4}$ & $\mathrm{R}$ & $6^{4}$ & $\mathrm{R}$ \\
\hline Tobramycin & 2 & $\mathrm{~s}$ & - & - & - & - \\
\hline Tigecycline & - & - & 0.25 & 1 & 0.25 & 1 \\
\hline Clarithromycin & $\leq 0.25$ & $\mathrm{~s}$ & 0.25 & $\mathrm{~s}$ & 1 & $\mathrm{~S}$ \\
\hline \multicolumn{7}{|c|}{$\begin{array}{l}{ }^{1} \text { No CLSI MIC breakpoints have been established (17). } \\
{ }^{2} \text { Imipenem MIC interpretation is not recommended by CLSI because of } \\
\text { difficulties with reproducibility of results (17). } \\
{ }^{3} \text { Meropenem MICs were interpreted using bacterial CLSI breakpoints that } \\
\text { are not standardized for mycobacteria. } \\
{ }^{4} \text { Kirby Bauer zone in millimeters. } \\
\text { MIC, minimum inhibitory concentration; Int, interpretation; } \\
\text { S, susceptible; R, resistant; I, intermediate; CLSI, clinical laboratory } \\
\text { standards institute. }\end{array}$} \\
\hline
\end{tabular}

Table 2 


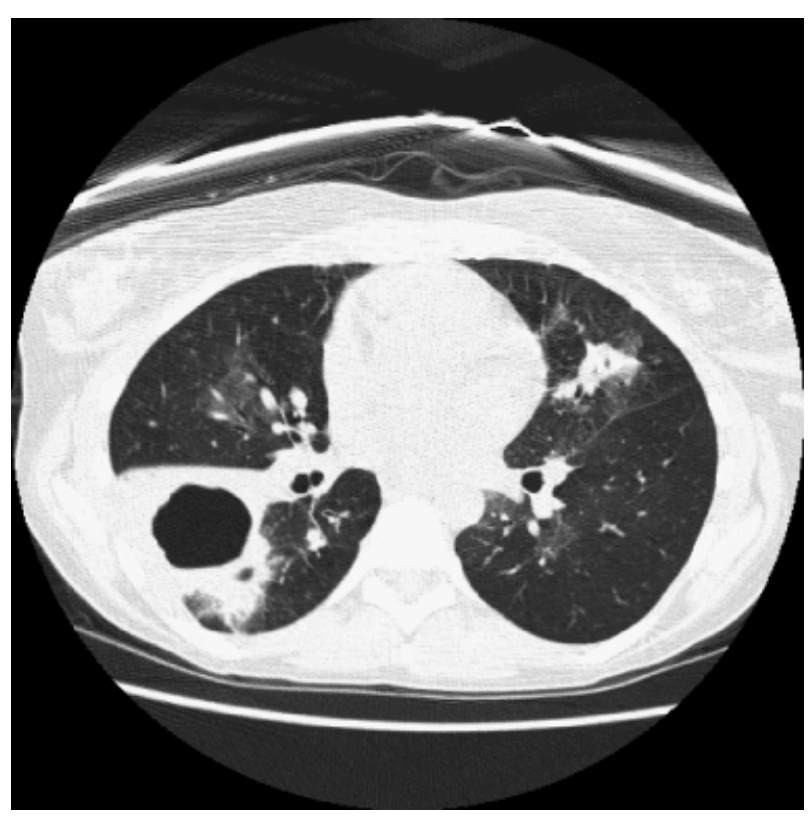

Fig. 4. Chest computed tomography scan in January 2008 demonstrating improvement in left upper lobe disease and worsening of infection in right upper lobe around a previous cavity.

the lung. The poor response to agents typically used to treat this organism, as well as the development of resistance despite the use of combination therapy, are unusual features. The clinical response to treatment with tigecycline suggests that this agent could be a useful component of therapy for this organism.

NTM such as $M$. chelonae are ubiquitous in nature and propagate in water, soil, and dust (9). Infection often occurs as a result of inhalation of organisms or ingestion of contaminated food or water. In immune competent individuals, the organisms are often eliminated through a functional immune response. In patients with underlying structural lung disease or immune compromise, partic-

Flow cytometry after discontinuation of ECP therapy

\begin{tabular}{|c|c|c|c|}
\hline Marker & Description & $\begin{array}{l}\% \text { of } \\
\text { lymphocytes }\end{array}$ & $\begin{array}{l}\text { Absolute cell } \\
\text { count (cells } / \mu \mathrm{L})\end{array}$ \\
\hline $\mathrm{CD} 3$ & Tcell & $52.2 \mathrm{~L}(59-79)^{1}$ & $1778(822-1902)$ \\
\hline CD4 & T-helper/inducer & $26.1 \mathrm{~L}(36-52)$ & $888(517-1169)$ \\
\hline $\mathrm{CD} 8$ & $\begin{array}{l}\text { T-suppressor/ } \\
\text { cytotoxic }\end{array}$ & $24.8(19-29)$ & 844 H (255-649) \\
\hline CD16,56 & Natural killer cell & 36 & 1226 \\
\hline CD19 & B cell & $11.5(3-13)$ & $392 \mathrm{H}(1-354)$ \\
\hline CD4:CD8 & $\mathrm{T} 4 / \mathrm{T} 8$ ratio & $1.05 \mathrm{~L}(1.2-2.6)$ & \\
\hline
\end{tabular}

Table 3 ularly with T-cell deficits, invasive infection can occur. Surprisingly, NTM are uncommon pathogens in patients undergoing HSCT (2). Among patients who undergo HSCT, particular risk factors for NTM that have been identified include allogeneic transplants, T-cell depleted SCTs, therapy for GVHD, and certain conditioning chemotherapeutic regimens $(1,10,11)$. A case series from Hong Kong demonstrated a higher incidence of NTM infections in unrelated allogeneic HSCT recipients $(1.9 \%)$ as compared with autologous SCTs (0\%) (10). The use of umbilical cord blood, unrelated mismatched donors, and additional immunosuppressive medications may have contributed to the higher incidence in this study.

Weinstock et al. (1) have reported the highest overall incidence of NTM infection to date in HSCT of $9.7 \%$ in patients who received T-cell depleted SCTs. Only $2.9 \%$ of the total cohort, however, had pulmonary infection (1). Others had previously reported much lower incidence rates of $1 \%$ or less overall $(2-4,12)$. In recent years an increased number of NTM cases have been reported in the literature and the incidence in general is thought to be increasing $(5,13)$. Increased patient survival, increased awareness of NTM, and enhanced diagnostic capabilities may be factors in this perceived increase. The most recent evaluation of the incidence of NTM infections was performed at New YorkPresbyterian Hospital where proven respiratory infection in non-human immunodeficiency virus-infected individuals was estimated at 2 cases/100,000 (14).

The diagnosis of NTM lung disease can often be difficult and requires a high index of suspicion. Distinguishing between colonization and true infection with NTM can be challenging. Symptoms of NTM infection are often nonspecific and include fever, cough, sputum production, and hemoptysis (6). If patients are immune suppressed to a significant degree or have persistent symptoms, the initial evaluation of patients who have undergone SCT should consist of a chest x-ray followed by chest CT. Findings on CT that may be characteristic for NTM infection include nodules ranging from $<3 \mathrm{~mm}$ to $>1 \mathrm{~cm}$, cavitation, bronchiectasis, consolidation, and bronchial wall thickening (15). If such findings are identified on imaging, the next step in evaluation would include either 3 or more sputum specimens sent for AFB smear and culture, or bronchoscopy with BAL specimens sent for AFB smear and culture with or without transbronchial biopsy. Other more common infectious etiologies, such as bacterial, fungal, or viral pathogens, must concomitantly be ruled out. Among NTM, $M$. chelonae is classified as a rapid grower, as it usually is isolated on selective media within 7 days of inoculation.

Often, NTM isolated from single sputum or BAL specimens without other characteristic findings represent contamination. Of $24 \mathrm{M}$. chelonae pulmonary isolates recovered at MD Anderson in Texas, only 8 (33\%) were consid- 
ered to represent true infection (6). In the New York-Presbyterian Hospital study, 24\% of pulmonary isolates met American Thoracic Society (ATS) criteria for true infection (14). Therefore, the following criteria have been established by the ATS and the Infectious Diseases Society of America to diagnose active infection with NTM (8):

(1) Clinical criteria - respiratory symptoms combined with a chest x-ray or chest CT that demonstrates nodular or cavitary opacities, or multifocal bronchiectasis with multiple small nodules and the exclusion of other diagnoses such as invasive fungal infection or bacterial infection.

(2) Microbiologic criteria - positive culture results from 2 sputum samples or positive culture results from a BAL or a biopsy of lung tissue demonstrating histological changes consistent with AFB infection combined with a positive culture from sputum or BAL. The presence of unusual NTM organisms needs to be considered carefully in clinical context because of the risk of contamination. Treatment considerations need to be individualized. If the above clinical and microbiologic criteria are not met, then an alternative diagnosis may need to be considered.

Resistance testing for NTM such as M. chelonae is recommended and can be performed at reference laboratories such as the University of Texas Health Center and National Jewish Hospital. Such testing is considered critical for selecting appropriate therapy by most experts (16). Unfortunately, minimum inhibitory concentration (MIC) clinical cutoffs are not available for certain agents such as imipenem-cilastin and tigecycline (17).

Treatment of pulmonary infection with NTM can be difficult. Most authors recommend using a regimen that includes clarithromycin plus 1 or more other active agents (based on susceptibility data) for up to 12 months until negative follow-up sputum cultures and radiographic improvement is documented $(8,18)$. Others have recommended the use of imipenem-cilastin and tobramycin in combination for initial therapy (7). Organisms such as M. abscessus and $M$. chelonae can develop resistance, particularly to macrolides (19), and therefore require treatment with at least 2 active agents. Both of these organisms are resistant to most agents used to treat Mycobacterium tuberculosis. The majority of $M$. chelonae isolates are sensitive to clarithromycin, tobramycin, and linezolid (8). Linezolid has demonstrated in vitro activity and has been used to successfully treat disseminated disease $(20,21)$. Fifty to $60 \%$ of isolates are sensitive to amikacin and imipenem-cilastin (8), although imipenem-cilastin MICs are not considered to be reliable by most experts, and are difficult to interpret because of difficulties with reproducibility owing to the prolonged incubation required $(16,17)$. Respiratory fluoro- quinolones have also been used in combinations with other drugs. In patients who do not respond to medical therapy, surgical resection may need to be considered. Without prospective trials, the optimal treatment of $M$. chelonae pulmonary infection remains unknown.

Tigecycline, an intravenous glycylcycline antibiotic, has in vitro activity against $M$. chelonae in addition to $M$. $a b$ scessus and Mycobacterium fortuitum (22), although clinical data is lacking. In the presented case, the patient's isolate retained susceptibility to tigecycline with a relatively low MIC of $0.25 \mu \mathrm{g} / \mathrm{mL}$. A tigecycline MIC $<2 \mu \mathrm{g} / \mathrm{mL}$ is generally interpreted as susceptible for most organisms. No standards currently exist to interpret MICs to tigecycline for mycobacteria, however. Given that the tigecycline $\mathrm{AUC}_{0-24 \mathrm{~h}}$ is $4.70 \mu \mathrm{g} \bullet \mathrm{h} / \mathrm{mL}(23)$, a clinical response to therapy would be predicted in the presented case. In addition, this class of drug is well distributed into tissues in high concentration. Glycylcyclines are active against organisms that are resistant to tetracyclines because of a modification of the molecule's D-ring, which prevents it from being affected by the efflux pumps and ribosomal changes that lead to tetracycline resistance (24). After each 4- to 6-week course of treatment with tigecycline, the patient seemed to respond clinically, although a significant radiological response was not observed. Therefore, tigecycline may be a useful agent combined with other active drugs to treat $M$. chelonae infection, although further large-scale randomized studies would need to be performed to determine true effectiveness. Because of the uncommon nature of NTM infection, it is unlikely that such a study will be performed.

GM-CSF has been used as an adjunctive agent in NTM infections, particularly for $M$. avium complex, to enhance immune surveillance given the poor response to antimicrobial treatment in many immune compromised individuals. Use of this growth factor induces granulocyte, monocyte, and macrophage function, all of which are important components of the immune response to NTM (25). Additional attempts at reducing immune suppression in the reported case included the use of ECP to substitute for calcineurin inhibitors and corticosteroids for the management of GVHD, as recent reports have suggested that ECP does not have effects on cell-mediated immunity (26). Prostaglandin $\mathrm{E}_{2}$ is locally immune suppressive and production of this cytokine is known to be stimulated by infection with mycobacteria (27-29). In an effort to further enhance local immune responses, an NSAID was administered to decrease production of prostaglandin $\mathrm{E}_{2}$; however, this had little discernable clinical impact on disease progression.

Outcomes to treatment are difficult to assess overall. In one study from MD Anderson in Texas, out of 8 patients with underlying cancer diagnoses with documented M. chelonae pneumonia, 2 survived, 2 demonstrated clinical improvement but died from their underlying malignancy, 
2 died with progressive pneumonia (1 of whom refused treatment), and 2 others died rapidly from their underlying malignancy, so their response to therapy could not be determined (6). Therefore, response to treatment in this study was difficult to determine because of the progression of the patients' underlying malignancies. In an earlier study that summarized responses to treatment in mostly nonimmunocompromised hosts in the era before the use of clarithromycin, mortality was $17 \%$ (30). Most patients in this group received cefoxitin and amikacin. The combination of cefoxitin and ciprofloxacin was also reported to be successful in one patient.

In summary, M. chelonae lower respiratory tract infection complicating SCT is rare. Diagnosis relies heavily on characteristic radiographic findings and the repeated isolation of the organism from sputum or BAL specimens. Combination antimicrobial therapy based on susceptibility testing is considered standard, with macrolides, tobramycin, linezolid, imipenem-cilastin, and amikacin as the most active agents. In cases of clinically significant pulmonary infection, one approach to initial treatment is to administer 4-6 weeks of intravenous agents followed by 12 months or more of oral agents until clinical and radiographic evidence of response is documented. Progressive disease and significant mortality has been reported in patients with immune-compromising underlying co-morbidities, such as HSCT, despite treatment. Elimination of immune suppressive treatments, adjunctive therapy with GM-CSF, and prostaglandin $\mathrm{E}_{2}$ manipulation, along with prolonged treatment with multiple antimicrobial agents to which the reported patient's isolate was susceptible, had no effect on the overall course of her disease. In the patient reported, the use of tigecycline appeared to be of clinical benefit.

\section{Acknowledgement:}

We would like to acknowledge Carrie Lapham for her assistance in preparing this manuscript.

\section{References}

1. Weinstock D, Feinstein M, Sepkowitz K, Jakubowski A. High rates of infection and colonization by nontuberculous mycobacteria after allogeneic hematopoietic stem cell transplantation. Bone Marrow Transplant 2003; 31: 1015-1021.

2. Roy V,Weisdorf D. Mycobacterial infections following bone marrow transplantation: a 20 year retrospective review. Bone Marrow Transplant 1997; 19: 467-470.

3. Gaviria J, Garcia P, Garrido S, Corey L, Boeckh M. Nontuberculous mycobacterial infections in hematopoietic stem cell transplant recipients: characteristics of respiratory and catheter-related infections. Biol Blood Marrow Transplant 2000; 6: 361-369.
4. Cordonnier C, Martino R, Trabasso P, et al. Mycobacterial infection: a difficult and late diagnosis in stem cell transplant recipients. Clin Infect Dis 2002; 38: 1229-1236.

5. Field S, Cowie R. Lung disease due to the more common nontuberculous mycobacteria. Chest 2006; 129: 1653-1672.

6. Jacobson K, Garcia R, Libshitz H, et al. Clinical and radiological features of pulmonary disease caused by rapidly growing mycobacteria in cancer patients. Eur J Clin Microbiol Infect Dis 1998; 17: 615-621.

7. Kourbeti I, Maslow M. Nontuberculous mycobacterial infections of the lung. Curr Infect Dis Rep 2000; 2: 193-200.

8. Griffith D, Aksamit T, Brown-Elliott B, et al. An official ATS/IDSA statement: diagnosis, treatment, and prevention of nontuberculous mycobacterial diseases. Am J Respir Crit Care Med 2007; 175: 367-416

9. Falkingham J 3rd. Nontuberculous mycobacteria in the environment. Clin Chest Med 2002; 23: 529-551.

10. AuW, Cheng V, Ho P, et al. Nontuberculous mycobacterial infections in Chinese hematopoietic stem cell transplantation recipients. Bone Marrow Transplant 2003; 32: 709-714.

11. Kurzrock R, Zander A, Vellekoop L, Kanojia M, Luna M, Dicke K. Mycobacterial pulmonary infections after allogeneic bone marrow transplantation. Am J Med 1984; 77: 35-40.

12. Navari R, Sullivan K, Springmeyer S, et al. Mycobacterial infections in marrow transplant patients. Transplantation 1983; 36: 509-513.

13. Doucette K, Fishman J. Nontuberculous mycobacterial infection in hematopoietic stem cell and solid organ transplant recipients. Clin Infect Dis 2004; 38: 1428-1439.

14. Bodle EE, Cunningham JA, Della-Latta P, Schluger NW, Saiman L. Epidemiology of nontuberculous mycobacteria in patients without HIV infection, New York City. Emerg Infect Dis 2008; 14: 390-396.

15. Hazelton T, Newell J Jr, Cook J, Huitt G, Lynch D. CT findings in 14 patients with Mycobacterium chelonae pulmonary infection. Am J Roentgenol 2000; 175: 413-416.

16. De Groote M, Huitt G. Infections due to rapidly growing mycobacteria. Clin Infect Dis 2006; 42: 1756-1763.

17. NCCLS. Susceptibility testing of Mycobacteria, Nocardiae, and other aerobic Actinomyctes; approved standard. NCCLS 2003; M24 A.

18. Tartaglione T. Treatment of nontuberculous mycobacterial infections: role of clarithromycin and azithromycin. Clin Ther 1997; 19: 626-638.

19. Vemulapalli R, Cantey J, Steed L, Knapp T, Thielman N. Emergence of resistance to clarithromycin during treatment of disseminated cutaneous Mycobacterium chelonae infection: case report and literature review. J Infect 2001; 43: 163-168.

20. Brown-Elliott B, Wallace R Jr, Blinkhorn R, Crist C, Mann L. Successful treatment of disseminated Mycobacterium chelonae infection with linezolid. Clin Infect Dis 2001; 33: 1433-1434.

21. Wallace R Jr, Brown-Elliott B, Ward S, Crist C, Mann L, Wilson R. Activities of linezolid against rapidly growing mycobacteria. Antimicrob Agents Chemother 2001; 45: 764-767.

22. Wallace R Jr, Brown-Elliott B, Crist C, Mann L, Wilson R. Comparison of the in vitro activity of the glycylcycline tigecycline (formerly GAR936) with those of tetracycline, minocycline and doxycycline against isolates of nontuberculous mycobacteria. Antimicrob Agents Chemother 2002; 46: 3164-3167.

23. Wyeth Pharmaceuticals. Tigecycline package insert (data on file).

24. Testa R, Petersen P, Jacobus N, Sum P-E, Lee V,Tally F. In vitro and in vivo antibacterial activities of the glycylcycline class of semisynthetic tetracyclines. Antimicrob Agents Chemother 1993; 37: 2270-2277.

25. de Silva T, Cope A, Goepel J, Greig J. The use of adjuvant granulocyte-macrophage colony-stimulating factor in HIV-related disseminated atypical mycobacterial infection. J Infect 2007; 54: e207-e210. 
26. Marshall S. Technology insight: ECP for the treatment of GvHD - can we offer selective immune control without generalized immunosuppression? Nat Clin Pract Oncol 2006; 3: 302-314.

27. Adams L, Gillis T, Hwang D, Krahenbuhl J. Effects of essential fatty acid deficiency on prostaglandin E2 production and cell-mediated immunity in a mouse model of leprosy. Infect Immun 1997; 65: 1152-1157.

28. Bahr G, Rook G, Stanford J. Prostaglandin-dependent regulation of in vitro proliferative response to mycobacterial antigens of peripheral blood lymphocytes from normal donors and from patients with tuberculosis or leprosy. Clin Exp Immunol 1981; 45: 646-653.

29. Venkataprasad N, Shiratsuchi H, Johnson J, Ellner J. Induction of prostaglandin E2 by human monocytes infected with Mycobacterium avium complex - modulation of cytokine expression. J Infect Dis 1996; 174: 806-811.

30. Singh N,Yu V. Successful treatment of pulmonary infection due to Mycobacterium chelonae: case report and review. Clin Infect Dis 1992; 14: $156-161$. 